\title{
NEW POSSIBILITIES OF ENVIRONMENTALY FRIENDLY CLEANING METHOD BY LASER TECHNOLOGY
}

\author{
Lýdia Sobotová', Miroslav Badida' \\ 1 Katedra procesného a environmentálneho inžinierstva, Strojnícka fakulta, Technická univerzita v Košiciach, \\ Letná 9, 04200 Košice, Slovakia, e-mail: lydia.sobotova@tuke.sk, miroslav.badida@tuke.sk
}

Received: 2016.08 .15 Accepted: 2016.10 .08 Published: 2016.12.01

\begin{abstract}
Still deteriorating environment, requirements for reducing of production waste, requirements for the protection of the working environment, the formation of new technologies and materials, as well as the economic conditions are forcing manufacturers to use the environmentally friendly technology. The contribution deals with new progressive possibilities of cleaning products, raw materials and with the establishment of a minimum production waste. The research focuses on the possibilities of laser cleaning of materials, under defined technological conditions. In the contribution is presented the results of realized experiments in Technical University of Kosice, together created in cooperation with Trumpf Slovakia, s.r.o.
\end{abstract}

Keywords: corrosion, laser cleaning technology, material, environment.

\section{INTRODUCTION}

In various branches of industries we must lubricate or protect material surfaces, components, with various types of coatings. Equally, the important role as lubrication of material or paints, is also degreasing or coating removing from these metal surfaces.

Surface protection or surface cleaning in the mechanical industry play an important role in the protection of materials, in material processing or as a finishing treatment of surfaces, or is used in the maintenance of machinery parts and tools. By cleaning operations not only remove lubricant and coatings from surfaces, but also clean mechanical particles of rust, abrasion and dust. With the continuously deteriorating of environment, it is necessary to use such cleaning technologies of materials that have no negative impacts on the environment. Also the environment protection is encompassed by standards and decrees of REACH (Registration, Evaluation and Authorisation of Chemicals) [1].

\section{CLEANING TECHNOLOGIES FOR MATERIALS}

Cleaning technologies for materials have to be quick, clear and short. We can divide the these technologies according to various methods of practice into [2]:

- mechanical cleaning technologies,

- chemical cleaning technologies,

- progressive cleaning technologies.

Mechanical cleaning belongs to the most oldest technologies, as blasting, brushing. These operations are uasually joined with dust, waste and noise $[3,4,5,6]$.

The cleaning of metal materials by chemical method requires the using of various degreasers, chemicals, acids, which are very dangerous for the environment both in storage of new and pure chemicals, their utilised solutions and during of using of the chemicals joined with chemical vapors $[7,8]$,

Progressive cleaning technologies as ultrasonic cleaning technology, water jet and abrasive water jet technologies, dry ice cleaning technol- 
ogy, laser cleaning are based on the utilisation of the physical parameters. They enable to work more quickly, save the time, effectively and can clean accessible parts of machines and tools, reduce waste, which are very important requirements of producers $[7,9,10,11,12,13]$.

\section{LASER CLEANING TECHNOLOGY}

Surfaces are structured or cleaned for a specific purposeas. Laser processing can be used to remove or surface finish of tha material, workpieces, tools.

Progressive laser cleaning technology safely removes coatings, contaminants, oxides, rust, production residues and more without chemicals, solvents, abrasives, water and with minimum of dust. Cleaning with laser light is highly precise. Treatment can be applied to the exact area required, including 2D and 3D shapes and on-the-fly without the need for masking. In contrast to conventional cleaning methods, such as sandblasting or ice pellet blasting, laser cleaning offers the benefit of being very quiet with minimum of waste. In contrast to ultrasound cleaning, laser cleaning not needs the chemicals and water for concrete cleaning. The laser technology can easily be integrated online into many production processes in a way not possible with other options. Thus time, costs and environmental burdens can be reduced by completing previously outsourced work in-house.

Laser cleaning technology offers a highly selective, reliable, precise and safe cleaning method of removing layers of corrosion, pollution, unwanted paint, lubricants, other sur-

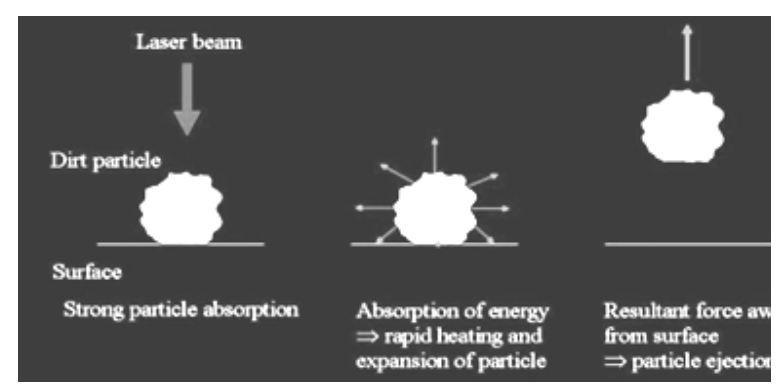

Fig. 1. Working of laser beam [13]

face coatings and are environmentally friendly, using no water or chemicals and producing no effluent. Primarily laser cleaning technology is used for industrial cleaning in the automobile, aerospace, bakery, food, electronics, restoration industries and surface treatment, renovation and paint removal applications. It also removes contaminants, production residue and coatings without damaging the substrate. Metallic and reflective surfaces are ideal although other substrates can be addressed. Laser cleaning systems offer an extremely high level of control and precision. The innovative laser cleaning systems combine power and versatility, with the lowest operating cost of all industrial cleaning methods. $[9,10,11,12]$.

Benefits of laser cleaning are:

- reduction of solvents (climate protection),

- reduction of abrasives and chemical cleaners,

- reduction of nuclear contaminated waste,

- reduction of process outsourcing burdens,

- reduction of masking materials, etc.,

- process can be used safely in the field (e.g. pylons in cropland) and locations when methods such as abrasive blasting are not possible.

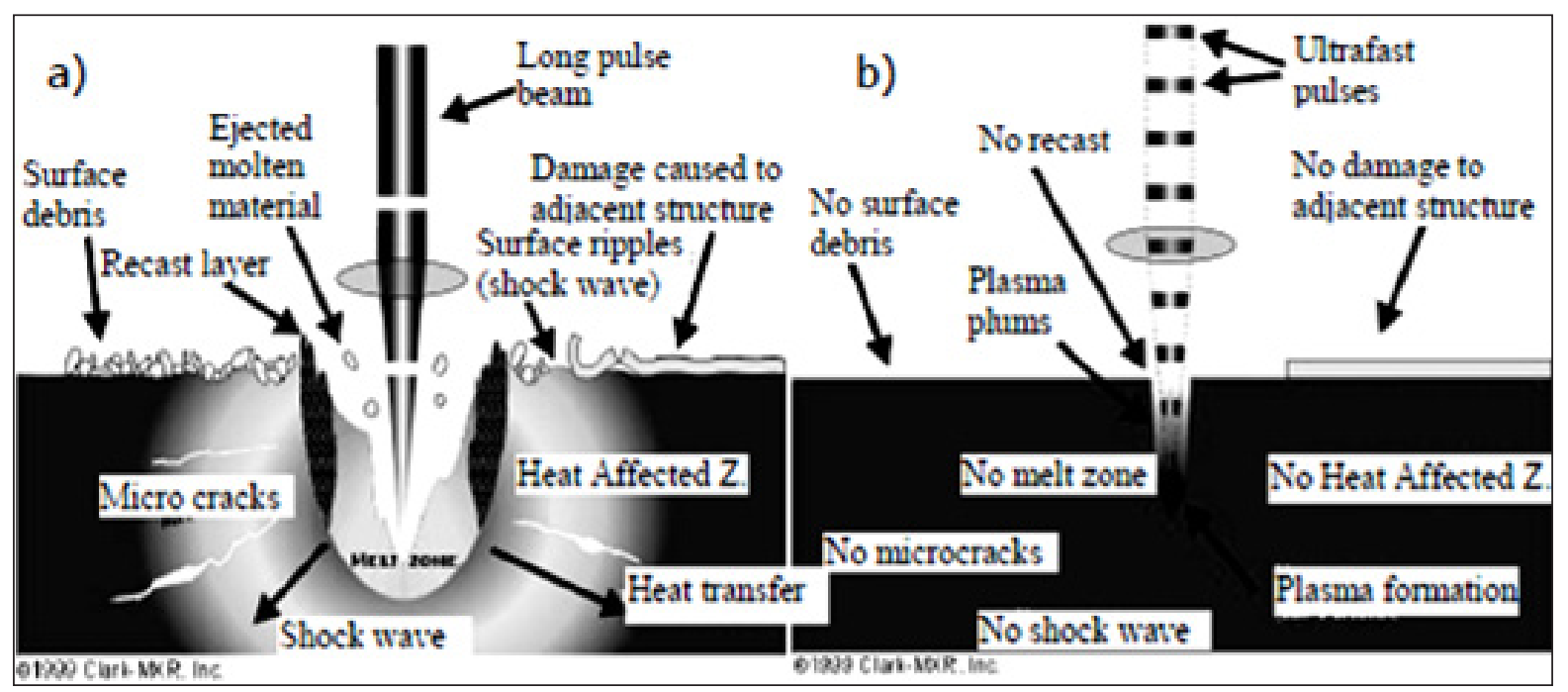

Fig. 2. Laser beam machining: a) by long pulses (15 ns), b) short pulses (150 fs) [13] 


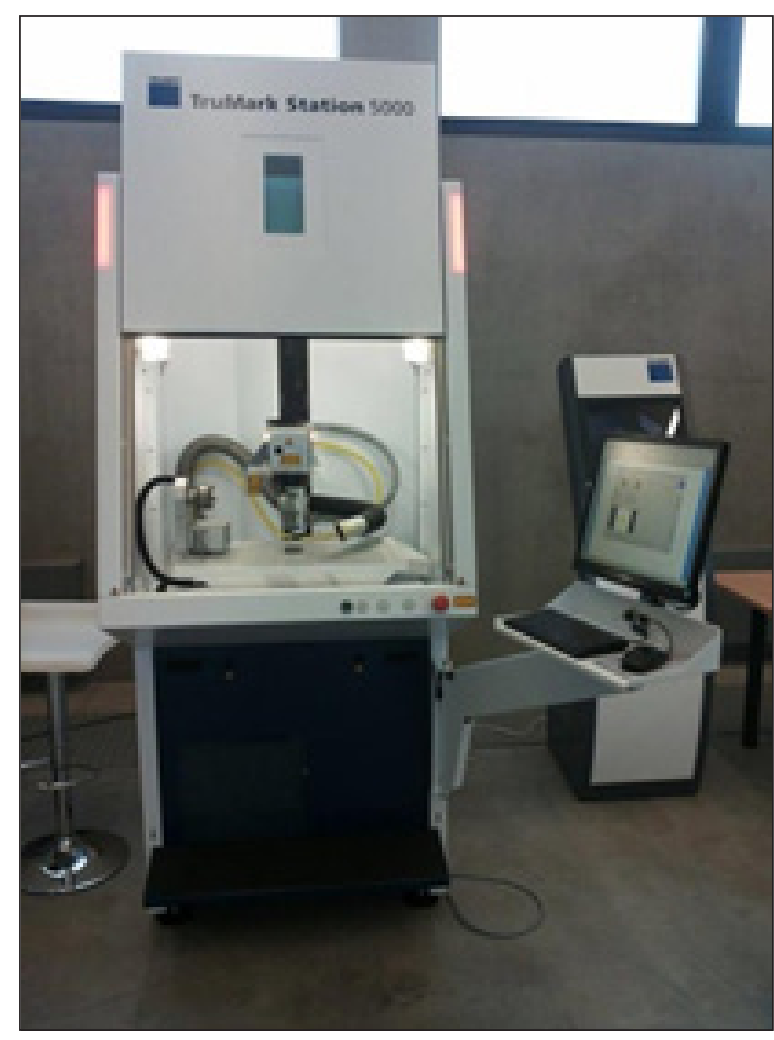

Fig. 3. The Trumpf Station 5000 [12]

Application of laser technique gives possibility of almost full control of the encrustation removal process at the surface of works. Selective and precise interaction of the light beam is a fundamental advantage of non-invasive treatment of more or less tightly connected unwanted surface layers.

The cleaning surface reflects laser energy and is minimally affected, however, any contaminants on the surface absorb the laser energy and are quickly vaporized, with any fumes or particulates removed by an in-built filter.

When a laser beam irradiates on the material surface, it may be considered that energy flows in only one direction in a semi- infinite body. In the Figure 1 is shown the principle of energy absorption of laser cleaning process.

The depth the laser energy penetrated into the material surface is constrained by the duration of the laser irradiation. Increasing irradiation time will allow the laser energy to penetrate deeper so as to raise the material substrate temperature. In the Figure 2 is shown the influence of the laser energy on the material.

The laser cleaning of material surface stops when the contaminant is consumed, and consequently there is a minimal heat build-up, avoid-

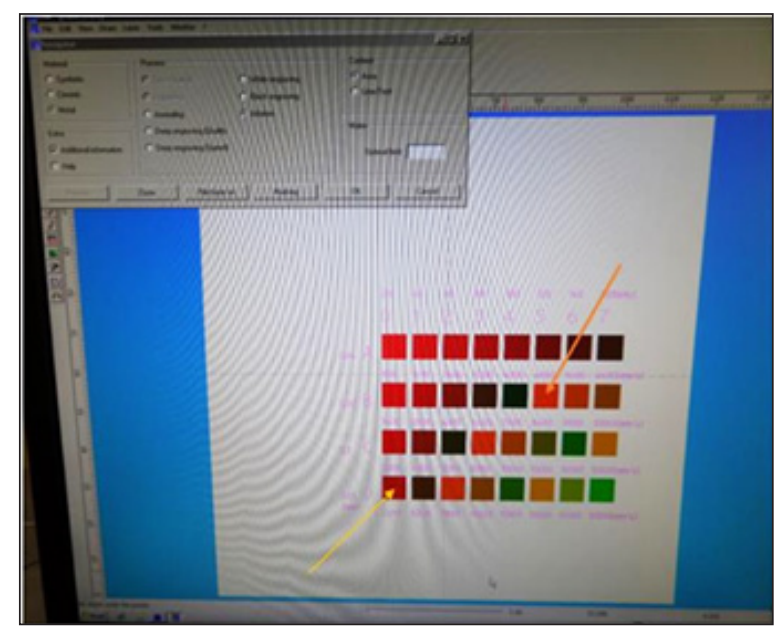

Fig. 4. Trumpf navigator program

ing the microscopic roughening and cracking of metal surfaces that can be present when using traditional cleaning methods $[9,11,12]$.

\section{Experimental testing and verification of laser cleaning technology}

The example of testing and workplace are shown in the Figure 3. In the experimental research the Trumpf Station 5000 was used, with software TruTops Mark and navigator shown in the Figure 4.

The condition of the laser parameters were: beam source TruMark 6130, optics $=$ F 163, wavelength $\lambda=1602 \pm 3 \mu \mathrm{m}$, speed of the laser beam [in $1000 \mathrm{~mm} / \mathrm{s}$ ], frequency [in $\mathrm{kHz}$ ] and focus of laser beam [in mm] were changed according to experimental tests.

Laser cleaning technology enables according of dirty of material to set up and to focus, the laser beam according to the requirement of material and process (Fig. 5).

For the realization of the experimental testing were prepared the following specimens from material steel sheet KOSMALT 190 IF (Table 1)

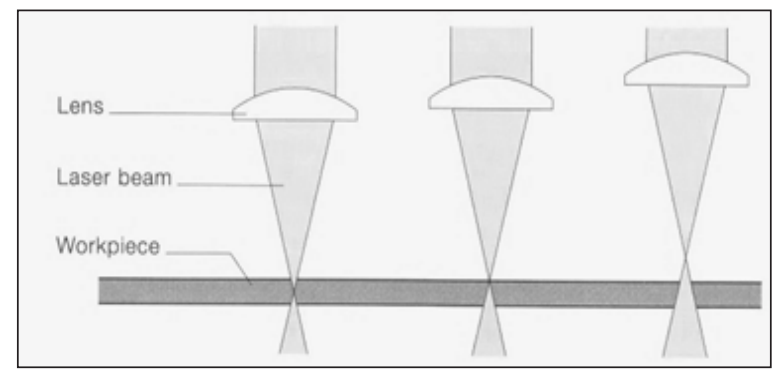

Fig. 5. Possibilities of set up of focus distance of laser beam [12] 
Table 1. The elements (mass concentration, \%) of material used in the experiment

\begin{tabular}{|l|c|c|c|c|c|c|c|}
\hline \multicolumn{1}{|c|}{ KOSMALT 190 IF, STN 038737} \\
\hline Element & $\mathrm{C}_{\max .}$ & $\mathrm{Mn}_{\max .}$ & $\mathrm{Si}_{\max .}$ & $\mathrm{P}_{\max .}$ & $\mathrm{S}_{\max .}$ & $\mathrm{Al}$ & $\mathrm{Cu}$ \\
\hline Content element (wt. \%) & 0,04 & 0,19 & 0,01 & 0,015 & 0,012 & $0,02-0,06$ & 0,06 \\
\hline
\end{tabular}

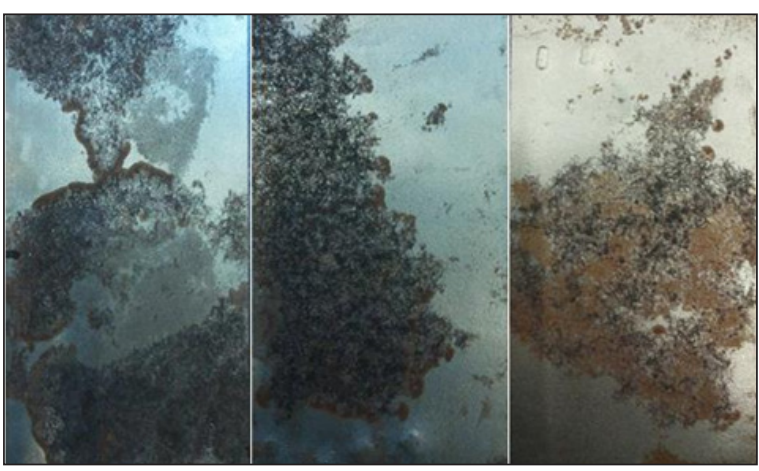

Fig. 6. Testing samples before laser cleaning, material Kosmalt 190 IF

with dimension $100 \times 50 \mathrm{~mm}$ and thickness $2 \mathrm{~mm}$, according to standard STN 038737.

The results after planned experiment, where the laser beam passed through the surface sample during the laser cleaning, are shown in the Figure 6 to Figure 14. For the each experiment were used corrosive samples on which were three fields, Fig.6. The laser beam crossed each testing cleaned fields from one to five times.

In the Figure 7 is shown the testing sample No.1 after cleaning operation, where the following technological parameters were used: pulse frequency: 28 [kHz], focus distance: $+2,0,-2$ $[\mathrm{mm}]$, feed speed of the beam: $2600[\mathrm{~mm} / \mathrm{s}]$. Laser beam passed through the sample five times.

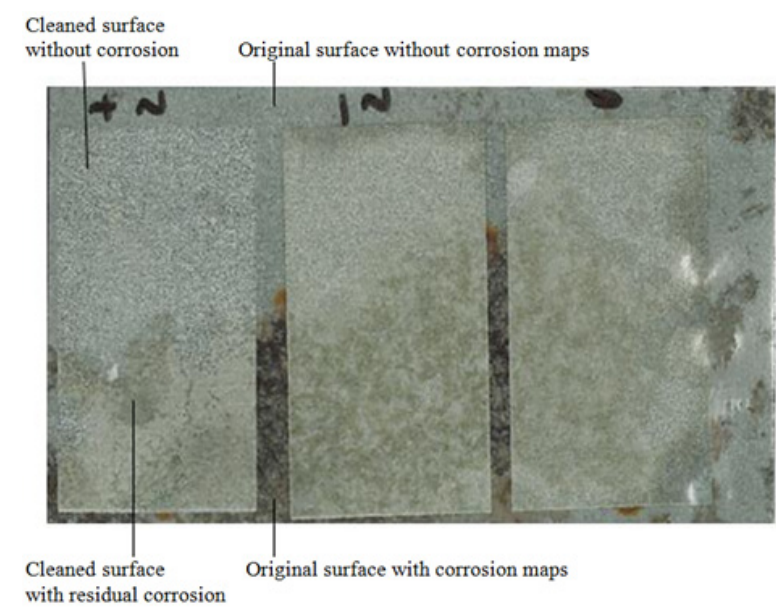

Fig. 7. Laser cleaned sample No.1 with focus distances: $0,-2,+2 \mathrm{~mm}$

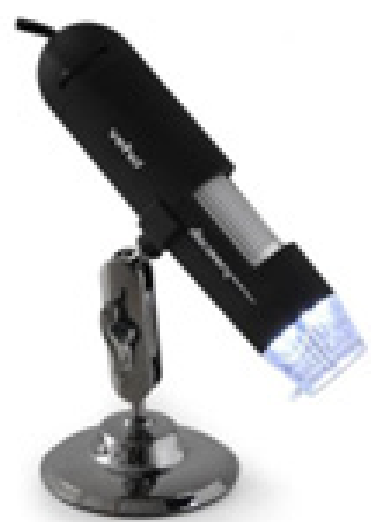

Fig. 8. USB microscope for quick visual evaluation

The best result os sample No.1 was obrained at the focus setting: +2 , but the maps of corrosion were still observed.

After laser claening the samples were observed visually and microscopically. The following Figue 8 shows the used USB microscope for quick visual evaluation, where we observed the lines in the tested material after laser cleaning and we could quickly and better compare the cleaned and non-cleaned part of the samples with the rest of corrosion, Figure 9.

Macrostructure and microstructure observations were completed at the microscope OLYMPUS, where were the microstructures with magnitude 50x and 100x.

In the Figure 10 and Figure 11 are shown the macrostructure of the sample No. 1, where is shown the border between cleaned and noncleaned places of the testing sample at the upper

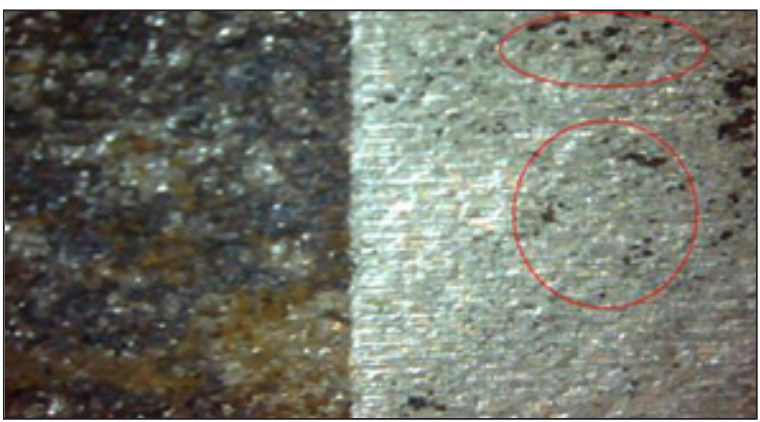

Fig. 9. Visual evaluation of tested sample No.1 with the cleaned and non-cleaned places, laser focus setting: 0 


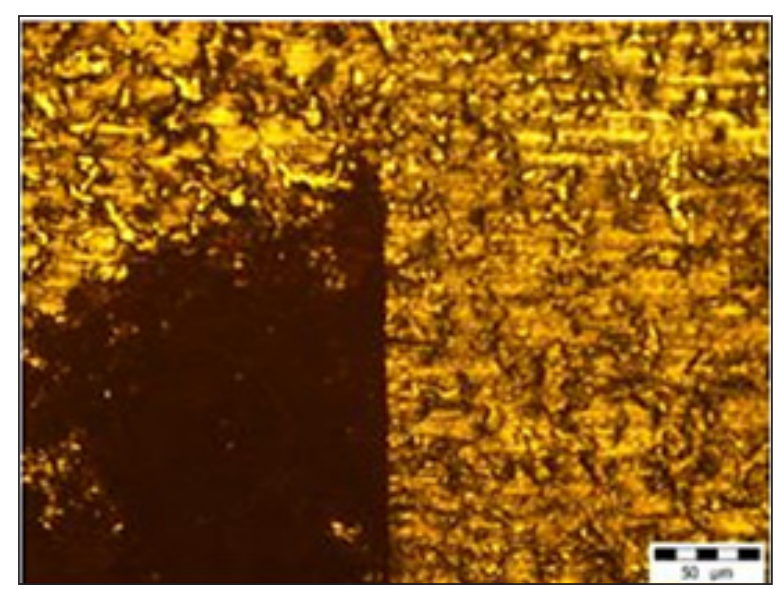

Fig. 10. Sample No. 1, magnitude 50×

defined technological parameters and magnitudes. The corrosion was removed from material. There is no observed the oriented structure, lines, because the pulse frequency was low.

The following microstructures Figure 12 and Figure 13 were prepared at magnitude $100 \times$ by light microscope at the focus distance of laser beam: $0 \mathrm{~mm}$. In the Figure 12 were used pulse frequency: $28 \mathrm{kHz}$, focus distance: $0,05 \mathrm{~mm}$, feed speed of the beam: 2600 $\mathrm{mm} / \mathrm{s}$. It is seen the maps of corrosion, but the sample is not oriented. In the Figure 13 were used higher pulse frequency $140 \mathrm{kHz}$ and feed speed of the beam: $8800 \mathrm{~mm} / \mathrm{s}$, where is shown the visible laser beam path, but in the tested area are visible the rest of corrosion maps on the tested material (dark places).

In the Figure 14 is shown the best cleared tested area, the pulse frequency $140 \mathrm{kHz}$ and feed speed of the beam: $9800 \mathrm{~mm} / \mathrm{s}$. The tested area has is better cleared, but some corrosion places were still marked.

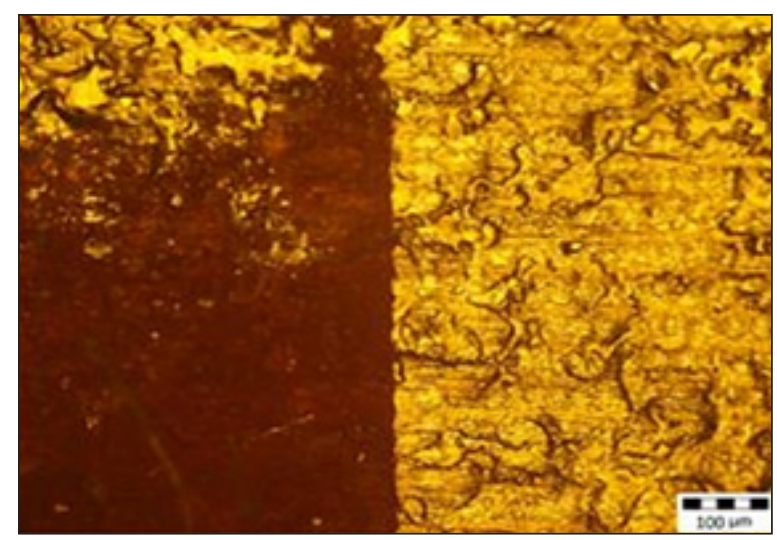

Fig. 11. Sample No. 1, magnitude 100×

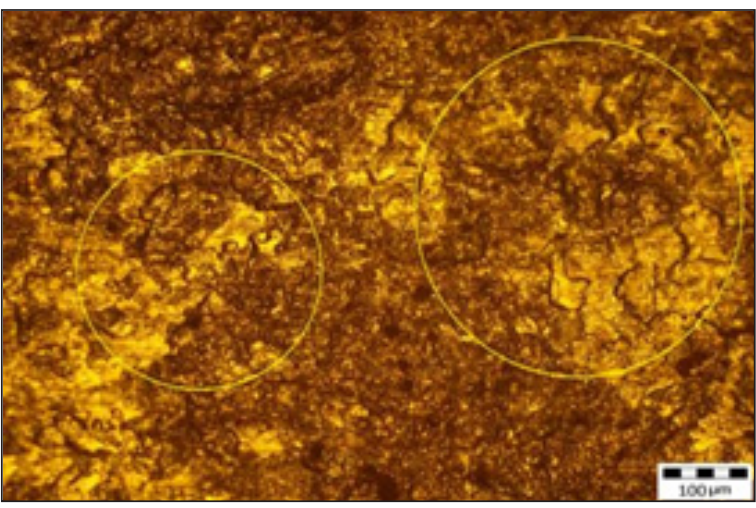

Fig. 12. Sample No.1 - middle part

\section{CONCLUSIONS}

Due to the ongoing problem of environmental degradation, we try to find ways how to minimize the impact of negative factors on the environment during the material cleaning. The industry as a whole is greatly contributes to environmental degradation. One of these ways was to try and to remove the rust from material surface without the using of chemicals and use the technolopgy with creating of minimum of waste.

In cooperation with TRUMPF Slovakia s.r.o., we tested and verified the possibility of cleaning of tested rust samples with a laser beam (with the compact machine TruMark 5000).

We changed the laser beam parameters and the result was the cleaned material surface at variou cnonditions. From the experimental results

From the experiments, we can make a conclusion after visual control and microstructure control as following $[8,9,10,11,12]$ :

1. The cleaning time is very short, some seconds, but also it depends on the magnitude of area.

2. Powerful, short, rapid, moving laser pulses produce micro-plasma bursts, shockwaves,

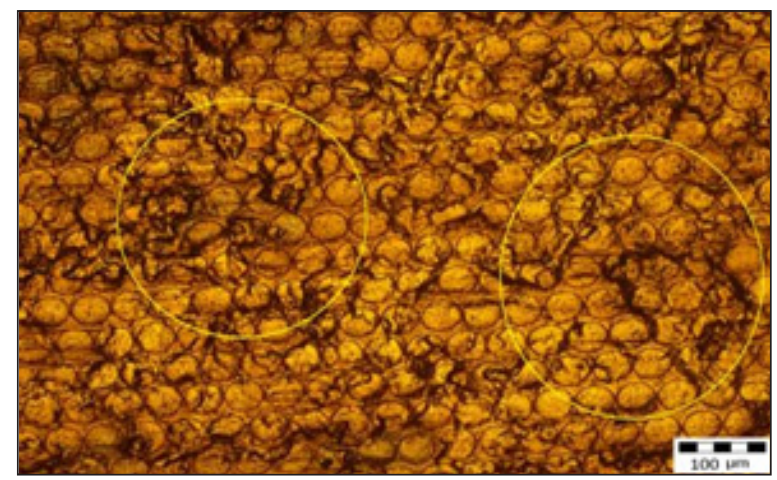

Fig. 13. Sample No.2 - middle part 


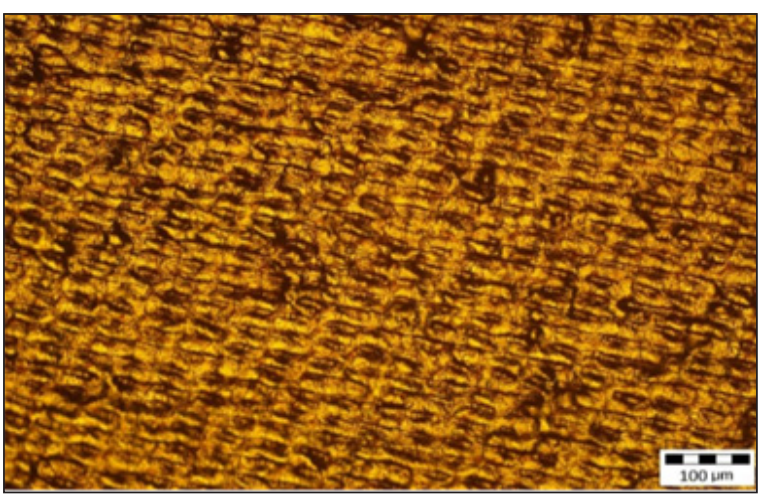

Fig. 14. Sample No.3 - middle part of cleaning place

thermal pressure resulting in sublimation and ejection of the target material.

3. We can clean the 2D and 3D surfaces, shaped surfaces, holes without problem.

4. During the cleaning of the surface it is necessary to prepare the cleaning program for programing of laser beam for each part and material individually.

5. Each material has own reflexibility, which is very important in laser technology.

6. Metallic and reflective surfaces are ideal although other substrates can be addressed. Optimized beam settings will not metallurgical change or damage the laser treated surface.

7. The quality of cleaned area depends on the setting of the technological parameters and parameters of laser beam, as pulse frequency, speed, focus of laser beam. A focused laser beam precisely vaporizes the target coating or contaminant.

8. Laser beam power density is accurately and easily adjusted to achieve cleaning results.

9. Laser cleaning systems offer an extremely high level of control and precision.

\section{Acknowledgements}

The work was supported by Ministry of Education of Slovak republic KEGA 048TUKE-4/2015.

\section{REFERENCES}

1. Badida M. et al. Basic of Enviromentalistics (in Slovak), TU Košice, Slovakia, 2013, 301.

2. Brezinová J. et al. Surface finishing in theory and practice (in Slovak), TU Košice, Slovakia, 2012, 250.

3. Badida M. et al. Hydroerosion and Environment. Lüdenscheid: Ram - Verlag, Germany, 2013. 131.

4. Dry ice cleaning. Cold Jet catalogue, 2014, www. coldjet.com.

5. Dry ice blasting without pellets. Cryo-clean snow. Linde, Slovakia 2014, www.lindeplastics.com

6. Dry ice cleaning Appliccations. Cryonimics, ARTIMPEX N.V., 2014, www.cryonomic.com

7. Ultrasonic Baths UR, US principle, 2015, www. retsch.com.

8. Kaščák L., Brezinová J. and Mucha J. Evaluation of corrosion resistance of galvanized steel sheets used in automotive production. Surface Engineering, 2014, Pfaffikon: Trans Tech Publication, 2015, 141-144.

9. Environmentally Friendly Cleaning with Light Reduces Costs (2005) PRWEB, www.prweb.com.

10. Laser cleaning. Preserve natural resources, 2015, www.cleanlaser.de.

11. Jackson M.J., Robinson G.M. and Chen X. Laser surface preparation of vitrified grinding wheels. ASM International, JMEPEG, 2006.

12. Cleaning with laser light. Clean-Lasersysteme GmbH, Germany, 2014. www.cleanlaser.de.

13. Laserové stroje/ Laser machines, 2014, TRUMPF Slovakia s.r.o., www.sk.trumpf.com.

14. Sobotová L. Badida M., Jenčo R. Environmental protection with using of new surface cleaning technologies. Albena, STEF92 Technology Ltd., 2015, 267-274. 